\title{
Study on Synthesis and Properties of Epoxy Phosphate
}

\author{
Cun Zhou ${ }^{1,2, a}$, Zilong Liu' 2, b \\ ${ }^{1}$ School of Environment and Chemical Engineering, Tianjin Polytechnic University, Tianjin 300387, \\ China \\ ${ }^{2}$ Tianjin Engineering Research Center of Textile Fiber Interface Treatment Technology, Tianjin \\ 300270, China \\ azhoucun588@126.com, bliuzilong6626@126.com
}

Keywords: epoxy resin, epoxy phosphate, interfacial tension, viscosity.

Abstract. An epoxy phosphate was successfully synthesized by reacting epoxy resin with phosphorus pentoxide. The chemical structure of epoxy phosphate was confirmed by using FT-IR. The interfacial tension and viscosity of aqueous solution of epoxy phosphate were tested. It is found that epoxy phosphate had good surface activity and its emulsion was pseudo plastic fluid.

\section{Introduction}

Epoxy resin had low shrinkage ratio, excellent adhesion, good stability, high mechanical strength, excellent electrical insulation and good processability ${ }^{[1]}$. It was widely used in the field of aerospace, electronic and electrical, national defense and military technology ${ }^{[2]}$. Epoxy resin was insoluble in water but soluble in organic solvents such as acetone. In recent years, due to the limitation of voganic compounds, the syn-thesis of water soluble epoxy resin become a hot topic ${ }^{[3]}$.

Phosphorus pentoxide was used as phosphorylation reagent to react with the epoxy resin by controlling the different reaction conditions. The surface activity of epoxy phosphate was examined and found to be excellent. The emulsion was pseudo plastic fluid.

\section{Experimental}

\subsection{Materials}

Epoxy resin (E51), Tianjin jindong chemical factory, IP; phosphorus pentoxide, CP ; $\mathrm{N}$-methylpyrrolidone $\mathrm{CP}$; potassium hydroxide $\mathrm{CP}$; acetone $\mathrm{CP}$.

\subsection{Modification reaction}

A three-necked flask equipped with thermometer and stirrer was charged with E51. $\mathrm{N}$-methylpyrrolidone were also added. The temperature was raised to $60^{\circ} \mathrm{C}$ until the resin was dissolved. Phosphorus pentoxide was then added for three times. The temperature was maintained at $60{ }^{\circ} \mathrm{C}$ for $4 \mathrm{~h}$ for the reaction to complete. Then some distilled water were added, they were $2 \%$ by weight of the epoxy phosphate. After the temperature was falled to $20^{\circ} \mathrm{C}$, potassium hydroxide was added into neutral. The reaction principle is shown in Fig. 1.

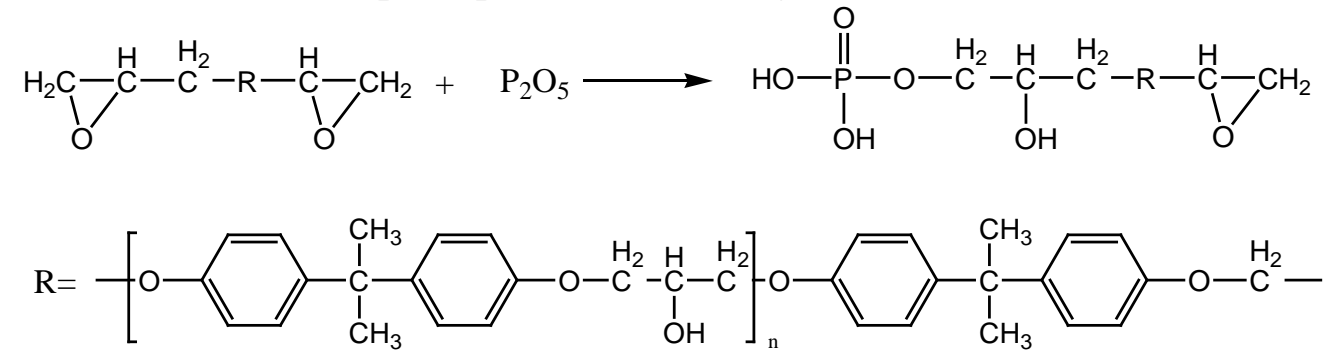

Fig. 1 Principle of the phosphorylation of epoxy resin 


\subsection{Emulsion preparation}

The temperature was raised to $40^{\circ} \mathrm{C}$ until the viscosity of resin decreased. Emulsion was carried out by dropping deionized water slowly to the resin with agitation.

\subsection{Separation product}

There were phosphate monoester, phosphate diester, epoxy resin, phosphoric and $\mathrm{N}$-methylpyrrolidone in the solvent. The product should be separated from the mixture. Acetone was used as extractive agent ${ }^{[4]}$, and the rest of the material was the product.

\subsection{Determination of infrared spectra}

The product was coated on the sodium chloride chip and the infrared spectrum of the product was determined.

\subsection{Test of aqueous solution of epoxy phosphate}

The epoxy phosphate was dissolved in different amounts of water. The interfacial tension and viscosity of emulsion were tested.

\section{Results and discussion}

\subsection{Infrared spectrum}

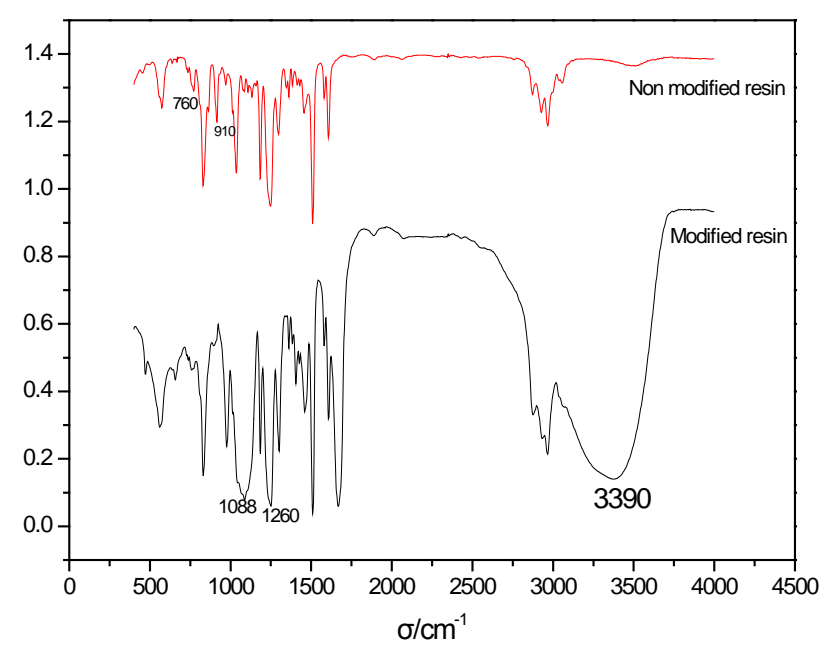

Fig. 2 FTIR spectrum of epoxy resin before and after reaction

The infrared spectra of the non modified epoxy resin and modified epoxy were shown in Fig .2. The absorption peak of epoxy group was at $910 \mathrm{~cm}^{-1}$ and $760 \mathrm{~cm}^{-1}$. But it disappeared after modifying ${ }^{[5]}$. The peak of P-O-C was at $1088 \mathrm{~cm}^{-1}$. The peak of $\mathrm{P}=\mathrm{O}$ was at $1260 \mathrm{~cm}^{-1}$. The peak of $3390 \mathrm{~cm}^{-1}$ was obviously enhanced, it was oxhydryl.

\subsection{Interfacial tension}

Test results of the interfacial tension were shown in Fig. 3.

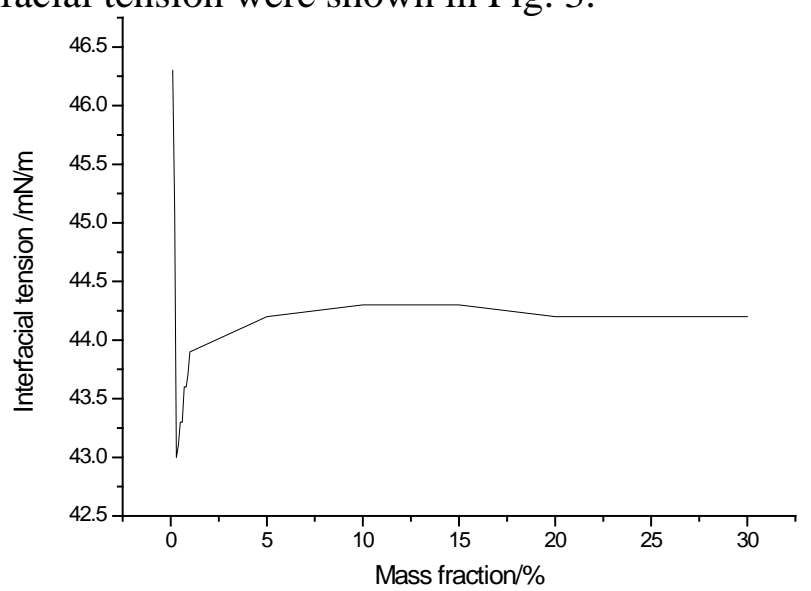

Fig. 3 The interfacial tension of emulsion with different mass fraction 
We can learn that the critical micelle concentration $(\mathrm{cmc})$ of the epoxy phosphate emulsion was $0.3 \%$ (mass fraction) in Fig. 3. When the concentration of the emulsion was increased to cmc, the surface tension of the gas-liquid increased to $44.2 \mathrm{mN} / \mathrm{m}$ slowly and remained stable.

\subsection{Test of viscosity}

Test conditions: rotor model CP52, $25^{\circ} \mathrm{C}$, shear rate $4 \mathrm{~s}^{-1}$, the viscosity of different mass fraction was shown in Fig. 4.

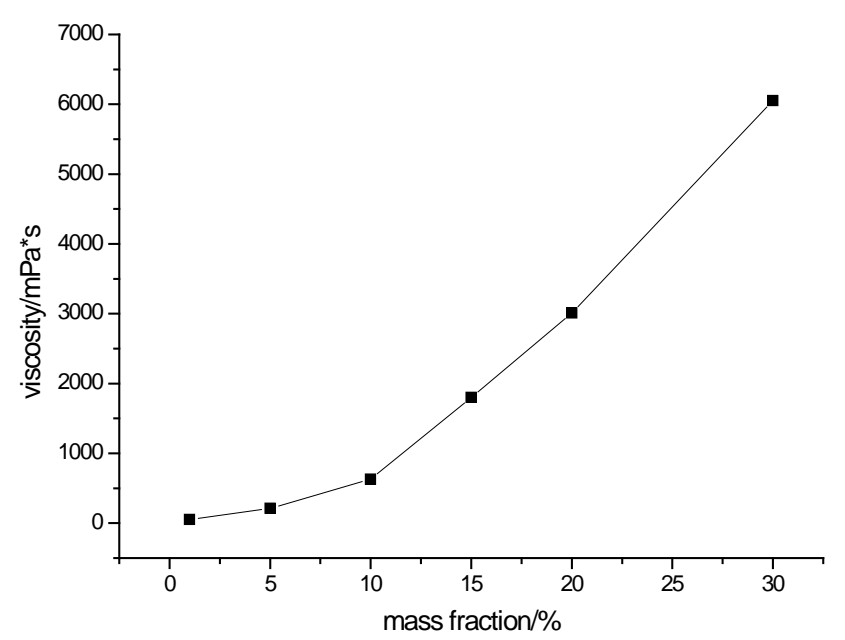

Fig. 4 Effect of different mass fraction on viscosity of emulsion

When the mass fraction was less than $10 \%$, the viscosity of the emulsion was very low ; when the mass fraction was more than $10 \%$, the viscosity of emulsion increased rapidly and the impact of mass fraction also increased accordingly .

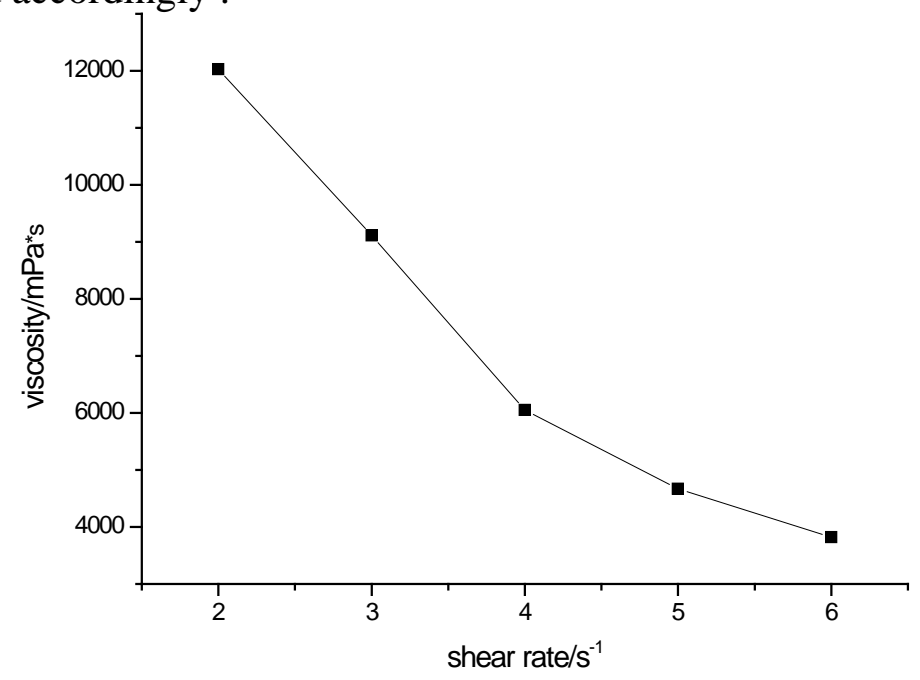

Fig. 5 Effect of shear rate on viscosity of emulsion

Fig. 5 shows the relationship between viscosity and shear rate. The viscosity of epoxy phosphate emulsion was decreased with increase of shear rate. The emulsion was shear thinning and pseudo plastic fluid ${ }^{[6]}$.

\section{Summary}

1. Epoxy phosphate is synthesized by reacting epoxy resin with phosphorus pentoxide.

2. The critical micelle concentration ( $\mathrm{cmc}$ ) of the epoxy phosphate emulsion is $0.3 \%$ (mass fraction).

3. The emulsion of epoxy phosphate is pseudo plastic fluid.

\section{References}

[1]Chen P , Liu S P , Wang D Z . Epoxy resin and its application[M]. Bei Jing: Chemical Industry Press , 2011,p. 1-13 
[2]Zhang Z Y, Huang Y H,Liao B,et a1. Studies on Particle Size of Waterborne Emulsion Derived from Epoxy Resin[J]. Eur. Polym. J. Vol. 37(2001) No. 6, p.1207-1211

[3]De Wet-R D , Knoetze J H , Cooray B , et al . Emulsion polymerization of an epoxy-acrylate emulsion stabilized with polyacrylate . J. Appl. Polym. Sci. Vol. 76(2000) No.3, p. 368-381

[4]Ding L , Wang GY, Hu C P , et al . Synthesis, Seperation and Characterization of the Water Dispersions of Graft Epoxy Resin[J]. J. Funct. Polym . Vol. 17(2004) No.2, p. 165-170

[5]Zheng W J , Ma C Y , Hu H P , et al . Discussion of the Mechanism of the Preparation and the Curing about Water-borne Epoxy Resin[J]. Polym Bull . Vol. (2006) No.1, p. 28-33

[6]Zhang Z Y , Huang Y H , et al . Waterborne Epoxy Resin by Chemical Modification[J] . Guang Zhou Chem . Vol. 25(2000) No.2, p. 7-11 